\title{
RECURRENCE RELATIONS FOR ORTHOGONAL POLYNOMIALS AND ALGEBRAICITY OF SOLUTIONS OF THE DIRICHLET PROBLEM
}

\author{
DMITRY KHAVINSON AND NIKOS STYLIANOPOULOS
}

Dedicated with admiration and in friendship to Professor Vladimir Maz'ya

\begin{abstract}
We show that any finite-term recurrence relation for planar orthogonal polynomials in a domain imply that the domain must be an ellipse. Our proof relies on Schwarz function techniques and on elementary properties of functions in Sobolev spaces.
\end{abstract}

\section{INTRODUCTION}

Let $\Omega$ be a bounded simply-connected domain in the complex plane, and let $\left\{p_{n}\right\}_{n=0}^{\infty}$ denote the sequence of Bergman orthogonal polynomials of $\Omega$. These are defined as the sequence

$$
p_{n}(z)=\gamma_{n} z^{n}+\gamma_{n-1} z^{n-1}+\cdots, \quad \gamma_{n}>0, \quad n=0,1,2, \ldots,
$$

of polynomials which are orthonormal with respect to the inner product

$$
\langle f, g\rangle:=\int_{\Omega} f(z) \overline{g(z)} d A(z),
$$

where $d A$ stands for the area measure. The associated $L^{2}$-norm is defined, as usual, by

$$
\|f\|_{L^{2}(\Omega)}:=\langle f, f\rangle^{\frac{1}{2}}=\left\{\int_{\Omega}|f(z)|^{2} d A(z)\right\}^{\frac{1}{2}} .
$$

Let $\Omega^{\prime}:=\overline{\mathbb{C}} \backslash \bar{\Omega}$ denote the complement (in $\overline{\mathbb{C}}$ ) of $\bar{\Omega}$ and let $\Phi$ denote the conformal map $\Omega^{\prime} \rightarrow \mathbb{D}^{\prime}:=\{w:|w|>1\}$, normalized so that, near infinity,

$$
\Phi(z)=\gamma z+\gamma_{0}+\frac{\gamma_{1}}{z}+\frac{\gamma_{2}}{z^{2}}+\cdots, \quad \gamma>0 .
$$

Note that the constant $1 / \gamma$ gives the (logarithmic) capacity cap $(\Gamma)$ of the boundary $\Gamma$ of $\Omega$ (see e.g. [19], [20]). The inverse conformal map $\Psi:=\Phi^{-1}$ :

Date: May 25, 2009.

1991 Mathematics Subject Classification. Primary 30C10; Secondary 47B32, 30C40, $31 \mathrm{~A} 25$.

Key words and phrases. Bergman orthogonal polynomials, Dirichlet's problem, recurrence relations. 
$\mathbb{D}^{\prime} \rightarrow \Omega^{\prime}$ has a Laurent series expansion of the form

$$
\Psi(w)=b w+b_{0}+\frac{b_{1}}{w}+\frac{b_{2}}{w^{2}}+\cdots,
$$

valid for $|w|>1$, where $b=1 / \gamma=\operatorname{cap}(\Gamma)$.

It is well-known that orthogonal polynomials with respect to any measure $\mu$ on the real line do satisfy a three-term recurrence relation, see e.g. [20]. By contrast, polynomials orthogonal with respect to the area measure, or the arc-length measure, in the complex plane $\mathbb{C}$, do not favor recurrence relations. To this end, Lempert [12] has produced examples of several, rather special domains, where the associated orthogonal polynomials do not satisfy ANY finite-term recurrence relation. M. Putinar and the second author have noted in [14] that the fact that "the Bergman polynomials of $\Omega$ satisfy a finite-term recurrence relation" is, actually, equivalent to the fact that "any Dirichlet problem in $\Omega$, with polynomial data, possesses a polynomial solution". The latter is the hypothesis of the so-called Khavinson-Shapiro conjecture [11], which states that only ellipses (or, ellipsoids in higher dimensions) have this property. This conjecture has attracted some attention and the reader is referred to [2], [15], [8], [10] and references therein, for results reporting on the recent progress in that direction. In [14] the authors showed that if the Bergman polynomials of a simply-connected domain $\Omega$ satisfy any finite-term recurrence relation and, in addition, the (necessarily algebraic) boundary of $\Omega, \partial \Omega \subseteq\{P(x, y)=0, P$ is a polynomial $\}$ satisfies the condition:

(B) the set $\{P=0\}$ is bounded in $\mathbb{C}$,

then $\Omega$ is an ellipse and the recurrence relation must be a three-term relation.

The main point of this note is to remove the assumption $(B)$. We do this, however, by assuming a finite-term recurrence of constant width, rather than one of variable width, as was the case in [14]. More precisely, we show that if the Bergman orthogonal polynomials of $\Omega$ satisfy a $(N+1)$-term recurrence relation, with $N$ a positive integer, then $\Omega$ is an ellipse and $N=2$. Yet, in order for our argument to work, it is not enough to assume that $\Omega$ is merely simply-connected, though a $C^{2}$-smooth Jordan boundary curve is sufficient. It remains an open question whether our results hold for any simply-connected domain. We strongly believe so, but we haven't been able to extend our proof to that case.

\section{MAIN RESUlts}

Let $\Omega$ be a bounded simply-connected planar domain and consider the Bergman space $L_{a}^{2}(\Omega)$ associated with $\Omega$. This is the Hilbert space of functions analytic and square integrable in $\Omega$. In this note we assume that the boundary $\Gamma$ of $\Omega$ is a Jordan curve. Under this assumption the Bergman polynomials $\left\{p_{n}\right\}_{n=0}^{\infty}$ of $\Omega$ form a complete orthonormal system in $L_{a}^{2}(\Omega)$ (see, e.g., [9] for weaker assumptions on $\Gamma$ regarding completeness in $L_{a}^{2}(\Omega)$ ). 
The standard way to construct the Bergman polynomials is by means of the Gram-Schmidt process. This is a linear algorithm that computes the sequence of the orthonormal polynomials recursively, by using as data the entrances of the complex moments matrix $H:=\left[\mu_{m, n}\right]_{m, n=0}^{\infty}$ of $\Omega$ :

$$
\mu_{m, n}:=\int_{\Omega} z^{m} \bar{z}^{n} d A(z) .
$$

It turns out that the complex moments matrix $H$ alone suffices to determine the (unique) sequence of Bergman polynomials of $\Omega$, and this determination is done, for each $p_{n}$, in a finite number of steps and by using a finite section of the moments matrix. (For more details regarding the general question of uniqueness properties of complex moments see [5].)

Clearly for all $n=0,1,2, \ldots$, we have

$$
z p_{n}(z)=\sum_{k=0}^{n+1} a_{k, n} p_{k}(z), \quad n=0,1, \ldots,
$$

where the Fourier coefficients $a_{k, n}$ are given by $a_{k, n}=\left\langle z p_{n}, p_{k}\right\rangle$. Then,

$$
\sum_{k=0}^{n+1}\left|a_{k, n}\right|^{2}<\infty, \quad n=0,1, \ldots
$$

The coefficients $a_{k, n}$ constitute the entrances of an infinite lower Hessenberg matrix $M$. This matrix is closely related to the multiplication operator by $z$ (the Bergman shift operator) $T_{z}: L_{a}^{2}(\Omega) \rightarrow L_{a}^{2}(\Omega)$, defined by $\left(T_{z} f\right)(z)=$ $z f(z)$, in the sense that $T_{z}$ can be represented with respect to the basis $\left\{p_{n}\right\}_{n=0}^{\infty}$ by $M$. Note that $T_{z}$ is linear and bounded on $L_{a}^{2}(\Omega)$.

Definition 2.1. We say that the Bergman polynomials $\left\{p_{n}\right\}_{n=0}^{\infty}$ satisfy a $(N+1)$-term recurrence relation for some fixed positive integer $N$, if for any $n \geq N-1$,

$$
z p_{n}(z)=a_{n+1, n} p_{n+1}(z)+a_{n, n} p_{n}(z)+\ldots+a_{n-N+1, n} p_{n-N+1}(z) .
$$

If the Bergman polynomials satisfy a $(N+1)$-term recurrence relation then one easily sees (cf. [14]) that the adjoint operator $T_{z}^{*}$ of the Bergman shift, and its multiples, increase the degree of a polynomial $p(z)$ subject to the constrain:

$$
\operatorname{deg}\left[\left(T_{z}^{*}\right)^{m} p\right] \leq m(N-1)+\operatorname{deg} p, \quad m \in \mathbb{N} .
$$

This follows easily, from the fact that $T_{z}^{*}$ can be represented with respect to the basis $\left\{p_{n}\right\}_{n=0}^{\infty}$ by the adjoint matrix $M^{*}$ of $M$ which, in this case, has an upper Hessenberg and banded form of constant width $N+1$.

The next result confirms the Khavinson-Shapiro conjecture (cf. [11], [2] and [15]) under an additional assumption on the degree of the polynomial solution to the Dirichlet problem. 
Theorem 2.1. Let $\Omega$ be a bounded simply-connected domain in $\mathbb{C}$ with a $C^{2}$ smooth Jordan boundary $\Gamma$, and assume that there exists a positive integer $N:=N(\Omega)$ with the property that the Dirichlet problem

$$
\left\{\begin{array}{l}
\Delta u=0 \text { in } \Omega, \\
u=\bar{z}^{m} z^{n} \text { on } \Gamma,
\end{array}\right.
$$

has a polynomial solution of analytic degree $\leq m(N-1)+n$ (in $z)$ and of conjugate analytic degree $\leq n(N-1)+m$ (in $\bar{z})$ for all positive integers $m$ and $n$. Then $\Omega$ is an ellipse and $N=2$.

Remark 2.1. By considering the polynomial $p(x, y)=\bar{z} z\left(=x^{2}+y^{2}\right)$ in $(2.7)$, it is easy to see that under the assumptions of Theorem 2.1, the boundary curve $\Gamma$ must be a part of the zero set of an algebraic polynomial and, hence, a piecewise analytic curve.

It is well-known that the Bergman polynomials of an ellipse satisfy a three-term recurrence relation. In fact, it is easy to check that they are suitably normalized Chebyshev polynomials of the 2 nd kind. The associated Hessenberg matrix in this case is triangular and goes by the name of Jacobi matrix. The following theorem states that this is the only possible case for an $(\mathrm{N}+1)$-recurrence to occur.

Theorem 2.2. With $\Omega$ and $\Gamma$ as in Theorem 2.1, assume that the Bergman orthogonal polynomials for $\Omega$ satisfy a $(N+1)$-term recurrence relation, with some $N \geq 2$. Then $\Omega$ is an ellipse and, hence, $N=2$.

We note that the conclusion of Theorem 2.2, for polynomials orthogonal with respect to the arc-length measure that satisfy a three-term recurrence relation (i.e. under the assumption $N=2$ ) goes back to P. Duren [7]. A similar result, as that of [7], but for polynomials orthogonal with respect to the harmonic measure on $\Gamma$, have been established in [6].

Theorem 2.1 becomes an easy consequence of Theorem 2.2, after we establish the equivalence between the assumptions of the two theorems. This latter task was essentially done in [14, Thm 1], under a more general definition for recurrences and, thus, without specific reference to the degree of the polynomial solution of (2.7). For our purposes here, however, we require the following explicit version of Theorem 1 of [14]:

Proposition 2.1. Let $\Omega$ be a bounded simply-connected domain in $\mathbb{C}$ with a $C^{2}$-smooth Jordan boundary $\Gamma$. Then, there exists a positive integer $N:=$ $N(\Omega)$, such that for all positive integers $m$ and $n$ the Dirichlet problem (2.7) with polynomial data $\bar{z}^{m} z^{n}$ has a polynomial solution of degree $\leq m(N-$ $1)+n$ in $z$, and $\leq n(N-1)+m$ in $\bar{z}$, if and only if the Bergman orthogonal polynomials for $\Omega$ satisfy a $(N+1)$-term recurrence relation.

The following result, which gives the ratio asymptotics for the Bergman polynomials, is needed in establishing Theorem 2.2. Its proof is a simple 
consequence of the strong asymptotics for Bergman polynomials over domains with smooth boundaries, established by P.K. Suetin in [19, Thm. 1.2] and, thus, we shall omit it.

Lemma 2.1. Assume that $\Omega$ is a bounded simply-connected domain in $\mathbb{C}$ with a $C^{2}$-smooth Jordan boundary $\Gamma$ and let $\left\{p_{n}\right\}_{n=0}^{\infty}$ denote the sequence of Bergman polynomials of $\Omega$. Then,

$$
\lim _{n \rightarrow \infty} \frac{p_{n+1}(z)}{p_{n}(z)}=\Phi(z), \quad z \in \overline{\Omega^{\prime}}
$$

We note, in passing, that strong asymptotics for Bergman polynomials were first derived by T. Carleman in [3], under the assumtion that $\Gamma$ is analytic.

Remark 2.2. For $\Omega$ simply-connected and bounded, a well-known result by Fejér asserts that the zeros of $p_{n}(z)(n \in \mathbb{N})$ are contained in $C o(\bar{\Omega})$, where $\operatorname{Co}(\bar{\Omega})$ denotes the convex hull of $\bar{\Omega}$. Under the additional assumption for $\Gamma$ in Lemma 2.1, it follows from [19, Thm 1.2] that there exists a positive integer $n_{0}$ such that the sequence $\left\{p_{n}\right\}_{n=n_{0}}^{\infty}$ has no zeros in $\Omega^{\prime}$.

Remark 2.3. Lemma 2.1 is precisely the reason we need to assume $C^{2}$ smoothness of $\Gamma$ in Theorem 2.1. Although we haven't been able to extend the ratio asymptotics to more general sets, we believe that (2.8) holds for arbitrary domains $\Omega$, such that $\Gamma=\partial \Omega=\partial \Omega^{\prime}$ is a continuum.

\section{Proofs}

Proof of Proposition 2.1. Fix two positive integers $m, n$ and assume that the Bergman orthogonal polynomials for $\Omega$ satisfy a $(N+1)$-term recurrence relation. Then, in view of $(2.6),\left(T_{z}^{*}\right)^{m} z^{n-1}=q(z)$, where $q$ is a polynomial of degree $\leq m(N-1)+n-1$. Therefore,

$$
\bar{z}^{m} z^{n-1}=q(z)+h(z), \quad z \in \Omega,
$$

where $h \in L^{2}(\Omega) \ominus L_{a}^{2}(\Omega)$. Let $Q(z)$ be a polynomial satisfying $Q^{\prime}=q$. According to the so-called Khavin's lemma (see e.g. [18, p. 26]) $h=\partial g$, with $g$ in the Sobolev space $W_{0}^{1,2}(\Omega)$. Integrating against $\partial$ we find,

$$
\bar{z}^{m} z^{n}=Q(z)+g(z)+\overline{f(z)}, \quad z \in \Omega,
$$

where $f \in L_{a}^{2}(\Omega)$. Since $\Gamma$ is smooth, it follows that $g=0$, a.e. on $\Gamma=\partial \Omega$, and thus,

$$
\bar{z}^{m} z^{n}=Q(z)+\overline{f(z)}, \quad \text { a. e. } z \in \Gamma,
$$

Moreover, from (3.9), Poincare's inequality and the smoothness of $\Gamma$, we infer easily (see, e.g., [1]) that $f$, in fact, belongs to the Hardy space $H^{2}(\Omega)$. (For the most up to date theory of Sobolev spaces we refer the reader to [13].) Similarly we have,

$$
\bar{z}^{n} z^{m}=G(z)+\overline{f_{1}(z)}, \quad \text { a. e. } z \in \Gamma,
$$


where $G$ is a polynomial of degree $\leq n(N-1)+m$, and $f_{1} \in L_{a}^{2}(\Omega) \cap H^{2}(\Omega)$. Hence,

$$
Q(z)+\overline{f(z)}=\overline{G(z)}+f_{1}(z), \quad z \in \Omega,
$$

and since $\Omega$ is simply-connected, we infer that

$$
Q(z)=f_{1}(z)+c \text { and } G(z)=f(z)+\bar{c}, \quad z \in \Omega,
$$

for some constant $c$. Hence, the Dirichlet problem in $\Omega$ with data $\bar{z}^{m} z^{n}$ has a polynomial solution whose analytic degree (in $z)$ is $\leq m(N-1)+n$, and its anti-analytic degree (in $\bar{z})$ is $\leq n(N-1)+m$.

For the converse assume that Dirichlet's problem for $\Omega$ with data $\bar{z}^{m} z^{n}$ has a polynomial solution $u(z)=Q(z)+\overline{G(z)}$, where $Q$ and $G$ are complex polynomials, with $\operatorname{deg}(Q) \leq m(N-1)+n$.

Let $h(z)$ be an bounded analytic function in $\bar{\Omega}$. Then, by Stokes' and Cauchy's theorems we obtain for $n \geq 1$ :

$$
\begin{aligned}
\left\langle\left(T_{z}^{*}\right)^{m} z^{n-1}, h\right\rangle & =\left\langle\bar{z}^{m} z^{n-1}, h\right\rangle=\int_{\Omega} \bar{z}^{m} z^{n-1} \overline{h(z)} d A(z) \\
& =-\frac{1}{2 n i} \int_{\Gamma} \overline{\zeta^{m}} \zeta^{n} \overline{h(\zeta)} d \bar{\zeta}=\frac{1}{n} \int_{\Omega} Q^{\prime}(z) \overline{h(z)} d A(z) \\
& =\langle q, h\rangle
\end{aligned}
$$

where $q(z):=Q^{\prime}(z) / n$. This implies that

$$
\left(T_{z}^{*}\right)^{m} z^{n-1}=q(z),
$$

where $\operatorname{deg}(q) \leq m(N-1)+n-1$ and, hence, the finite-term recurrence relation for the Bergman polynomials.

Proof of Theorem 2.2. Assume that the Bergman polynomials of $\Omega$ satisfy the recurrence relation (2.5) for some $N \geq 2$. Then, from Proposition 2.1 and Remark 2.1, we see that $\Gamma$ must be piecewise analytic.

Now we argue as in $[7$, p. 314]: For the moment, we assume that each of the $N+1$ sequences of the Fourier coefficients

$$
\alpha_{n}^{(1)}:=a_{n+1, n}, \quad \alpha_{n}^{(2)}:=a_{n, n}, \quad \ldots, \quad \alpha_{n}^{(N+1)}:=a_{n-N+1, n}, n \in \mathbb{N},
$$

is bounded, and then proceed as follows:

(i) divide both sides of $(2.5)$ by $p_{n}(z)$ (for $z \in \mathbb{C} \backslash C o(\bar{\Omega})$ );

(ii) take the limit as $n \rightarrow \infty, n \in \Lambda$, on both sides of the resulting equation, where $\Lambda$ is an appropriate subsequence of $\mathbb{N}$, chosen so that each sequence in (3.10) tends to a finite limit;

(iii) note that

$$
\frac{p_{n-k}}{p_{n}}=\frac{p_{n-1}}{p_{n}} \frac{p_{n-2}}{p_{n-1}} \cdots \frac{p_{n-k}}{p_{n-k+1}}, \quad k \leq N-1
$$

(iv) apply Lemma 2.1. 
The above steps yield that the inverse exterior conformal map $\Psi: \mathbb{D}^{\prime} \rightarrow \Omega^{\prime}$ has a finite Laurent expansion of the form

$$
\Psi(w)=b w+b_{0}+\frac{b_{1}}{w}+\frac{b_{2}}{w^{2}}+\cdots+\frac{b_{N-1}}{w^{N-1}}, \quad|w|>1 .
$$

To verify that all the sequences in (3.10) are bounded, one simply has to apply the Cauchy-Schwarz inequality, for $j=1,2, \ldots, N+1$ and $n \geq N-1$ :

$$
\begin{aligned}
\left|\alpha_{n}^{(j)}\right| & =\left|a_{n+2-j, n}\right|=\left|\int_{\Omega} \bar{z} p_{n+2-j}(z) \overline{p_{n}(z)} d A(z)\right| \\
& \leq\|z\|_{\infty}\left\|p_{n+2-j}\right\|_{L^{2}(\Omega)}\left\|p_{n}\right\|_{L^{2}(\Omega)}=\|z\|_{\infty},
\end{aligned}
$$

where $\|\cdot\|_{\infty}$ stands for the sup-norm on $\bar{\Omega}$.

From (3.11), it follows that $\Psi$ is a rational function. This implies that $\Omega^{\prime}$ is an unbounded quadrature domain, hence the associated Schwarz function $S(z)$, with $S(z)=\bar{z}$ on $\Gamma:=\partial \Omega=\partial \Omega^{\prime}$, has a meromorphic extension to $\Omega^{\prime}$, i.e.,

$$
S(z)=r(z)+\sum_{j=1}^{M} \sum_{l=1}^{k_{j}} \frac{c_{j, l}}{\left(z-z_{j}\right)^{l}}+f(z),
$$

where $z_{j} \in \Omega^{\prime}, k_{j} \in \mathbb{N}, r(z)$ is a polynomial of degree $d$, and $f(z)$ is analytic and bounded in $\Omega^{\prime}$, see e.g. [17].

We show first that all the constants $c_{j, l}, j=1, \ldots, M, l=1, \ldots, k_{j}$, in (3.12) vanish.

Let $P(z):=\prod_{j=1}^{M}\left(z-z_{j}\right)^{k_{j}}$ and consider the Dirichlet problem (2.7) with data $\bar{z} P(z)$. Our hypothesis and Proposition 2.1 imply that there exist analytic polynomials $h(z)$ and $g(z)$ such that

$$
\bar{z} P(z)=g(z)+\overline{h(z)}, \quad z \in \Gamma .
$$

(Note that $\operatorname{deg}(h) \geq 1$, otherwise on $\Gamma, \bar{z}=S(z)$ equals to a rational function and $\Gamma=\partial \Omega$ is a circle, according to a well-known theorem of P. Davis, see [4, p. 104].) Let $R(z)=\overline{S(z)}$ be the anti-conformal reflection about $\Gamma$. Obviously, by (3.12), $R(z)$ extends to $\Omega^{\prime}$ and has poles at $\infty$ and $\left\{z_{j}\right\}_{j=1}^{M}$. From (3.12) and (3.13) we see that on $\Gamma$,

$$
g(z)+\overline{h(R(z))}=r(z) P(z)+F(z),
$$

where $F(z)$ is analytic in $\Omega^{\prime} \backslash\{\infty\}$ and it may have a pole of order at most $\sum_{j=1}^{M} k_{j}$ at $\infty$. Since both sides of (3.14) are analytic functions of $z,(3.14)$ holds on any path originating on $\Gamma$ along which $S(z)$ continues analytically.

Now, let $\gamma$ be any path in $\Omega^{\prime} \backslash\{\infty\}$ joining $\Gamma$ to a given pole $z_{j}$ and avoiding all other poles. Then the right hand side of (3.14) stays bounded on $\gamma$ and so does $g(z)$, while $\overline{h(R(z))} \rightarrow \infty$ at $z_{j}$, because $|R(z)| \rightarrow \infty$ at $z_{j}$ and $h(z)$ is a (non-constant) polynomial . This is a contradiction and, therefore, $S(z)$ can have no finite poles in $\Omega^{\prime}$. That is,

$$
S(z)=r(z)+f(z), \quad z \in \Omega^{\prime}
$$


where $f(z)$ is analytic in $\Omega^{\prime}$ (including $\infty$ ).

Consider now the Dirichlet problem (2.7) with data $z \bar{z}=|z|^{2}$. In view of our hypothesis and Proposition 2.1, we can find a polynomial $g(z)$ of degree $k \geq 2$ (since if $k \leq 1$, then $\Gamma$ is obviously a circle and $N=1$ ), such that, for $z \in \Gamma$,

$$
2 \operatorname{Re}\{g(z)\}=g(z)+\overline{g(z)}=|z|^{2}=z S(z),
$$

or, using $R(z)$ and (3.15),

$$
g(z)+\overline{g(R(z))}=z r(z)+z f(z), \quad z \in \Gamma .
$$

Consider a path $\gamma$ in $\Omega^{\prime}$ joining $\Gamma$ to $\infty$. Since both sides of (3.17) are analytic in $\Omega^{\prime}$, (3.17) holds along $\gamma$. Yet, near $\infty$ we have $|g(z)| \sim|z|^{k}$, $|\overline{g(R(z))}| \sim|z|^{d k}$, and the right hand side of (3.17) behaves as $O\left(|z|^{d+1}\right)$, This can only be possible if $d k=d+1$, i.e. since $k \geq 2$, only if $d=1$ and $k=2$. From this it already, of course, follows that $\Omega$ is an ellipse and $N=2$. However, it may be worthwhile to point out the following observation as well. Thus,

$$
S(z)=c z+f(z), \quad z \in \Omega^{\prime}
$$

with $f$ analytic in $\overline{\Omega^{\prime}}$.

But this implies right away that $\Omega^{\prime}$ is a null-quadrature domain - cf. [17] and [18]. Indeed, using Green's and Cauchy's theorems we have for any number $m \geq 3$ :

$$
\begin{aligned}
\int_{\Omega^{\prime}} \frac{1}{z^{m}} d A(z) & =-\frac{1}{2 i} \int_{\Gamma} \frac{1}{z^{m}} \bar{z} d z=-\frac{1}{2 i} \int_{\Gamma} \frac{1}{z^{m}} S(z) d z \\
& =-\frac{1}{2 i} \int_{|z|=R} \frac{1}{z^{m}}\{c z+f(z)\} d z=0,
\end{aligned}
$$

for large enough $R$, since $f$ is analytic in $\Omega^{\prime}$ (including $\infty$ ). From this, in view of a theorem of Sakai [16, Thm 1], we infer that $\Omega^{\prime}$ must be the exterior of an ellipse. Hence $\Omega$ is an ellipse and, thus, $N=2$.

\section{Concuding Remarks}

We finish with a number of remarks.

(i) As we have pointed out above, the main place where the $C^{2}$-regularity of the boundary $\Gamma=\partial \Omega$ is needed was the application of the strong asymptotics for Bergman polynomials of Suetin [19], that yield Lemma 2.1. Moreover, it is clear from the proof of Theorem 2.2 that we only need (2.8) to hold on a continuum subset of $\Omega^{\prime}$, in a neighborhood of $\infty$. It looks quite plausible that, in this weaker form, (2.8) holds for arbitrary, bounded Jordan domains. Yet, we haven't been able to derive it for such general domains or, find a pertinent result in the literature.

(ii) For a most updated account on the status of the Khavinson-Shapiro conjecture in its full generality, mostly due to the work of H. Render [15], we refer the reader to the recent survey [10]. 
(iii) For a quite different approach, regarding singularities of solutions of the Dirichlet problem in $\mathbb{R}^{2}$, we refer the reader to [2], also cf. [8].

(iv) Finiteness of only the first row of the adjoint matrix $M^{*}$ associated with $T_{z}^{*}$, is not sufficient to yield Theorems 2.1-2.2, or Proposition 2.1 (unless, of course, $a_{0, n}=0, n \geq 2$, cf. [14, Prop. 1]). For example, take $\Gamma$ to be the bounded component of $\left\{x^{2}+y^{2}-1+\epsilon\left(x^{3}-3 x y^{2}\right)=0\right\}$, where $\epsilon>0$ is small enough so that $\Gamma$ is a perturbation of the unit circle. Then, the quadratic data $z \bar{z}$ are matched on $\Gamma$ by a cubic harmonic polynomial, despite the fact that $\Gamma$ is not an ellipse.

(v) The assumption that $\Omega$ is simply-connected is not really necessary. As is seen from the arguments in [2], the hypothesis in the Khavinson-Shapiro conjecture implies that $\Omega$ is simply-connected.

(vi) The condition that a finite-term recurrence relation (of some constant width $N+1$ ), satisfied by the Bergman polynomials of $\Omega$, is stronger than the hypothesis of the Khavinson-Shapiro conjecture for $\Omega$. This is so because the Khavinson-Shapiro conjecture does not involve any assumption on the degree of the polynomial solution. Thus a full proof of the conjecture is still amiss.

(vii) If the hypothesis of the Khavinson-Shapiro conjecture is satisfied then, clearly, $\Gamma=\partial \Omega$ is algebraic and, hence, piecewise analytic. Yet, in order to be able to use the ratio asymptotics for the Bergman polynomials, as they have been obtained by Suetin in [19], we must eliminate the possibility that $\Gamma$ has cusps. Perhaps, whenever the hypothesis of the KhavinsonShapiro conjecture holds (see Proposition 2.1) the cusps cannot occur a priori. We haven't been able to prove this either. We note however that it is possible to have cusped curves on which a quadratic matches a harmonic polynomial, e.g., $y^{2}=x^{3}-3 y^{2} x$.

Acknowledgement. Part of this work was carried out during the authors' respective visits to the University of Cyprus and the University of South Florida. The authors also, acknowledge, gratefully, support by the National Science Foundation (D.K.) and the University of Cyprus Research Committee (N.S.).

\section{REFERENCES}

1. R. A. Adams and J. J. F. Fournier, Sobolev Spaces, second ed., Pure and Applied Mathematics (Amsterdam), vol. 140, Elsevier/Academic Press, Amsterdam, 2003.

2. S. R. Bell, P. Ebenfelt, D. Khavinson, and H. S. Shapiro, On the classical Dirichlet problem in the plane with rational data, J. Anal. Math. 100 (2006), 157-190.

3. T. Carleman, Über die approximation analytisher funktionen durch lineare aggregate von vorgegebenen potenzen, Ark. Mat., Astr. Fys. 17 (1923), no. 9, 215-244.

4. P. Davis, The Schwarz Function and its Applications, The Mathematical Association of America, Buffalo, N. Y., 1974, The Carus Mathematical Monographs, No. 17.

5. P. Davis and H. Pollak, On the analytic continuation of mapping functions, Trans. Amer. Math. Soc. 87 (1958), 198-225.

6. A. A. Dovgoshei, Three-term recurrence relation for polynomials orthogonal with respect to harmonic measure, Ukrainian Math. J. 53 (2001), 167-177. 
7. P. Duren, Polynomials orthogonal over a curve, Michigan Math. J. 12 (1965), 313-316.

8. P. Ebenfelt, D. Khavinson, and H. S. Shapiro, Algebraic aspects of the Dirichlet problem, Quadrature domains and their applications, Oper. Theory Adv. Appl., vol. 156, Birkhäuser, Basel, 2005, pp. 151-172.

9. L. I. Hedberg, Approximation in the mean by analytic functions, Trans. Amer. Math. Soc. 163 (1972), 157-171.

10. D. Khavinson and E. Lundberg, The search for singularities of solutions to the Dirichlet problem: recent developments, preprint: http://shell.cas.usf.edu/ ${ }^{\sim}$ dkhavins/publications.html.

11. D. Khavinson and H. S. Shapiro, Dirichlet's problem when the data is an entire function, Bull. London Math. Soc. 24 (1992), no. 5, 456-468.

12. L. Lempert, Recursion for orthogonal polynomials on complex domains, Fourier analysis and approximation theory (Proc. Colloq., Budapest, 1976), Vol. II, Colloq. Math. Soc. János Bolyai, vol. 19, North-Holland, Amsterdam, 1978, pp. 481-494.

13. V. G. Maz'ya, Sobolev Spaces, Springer Series in Soviet Mathematics, Springer-Verlag, Berlin, 1985, Translated from the Russian by T. O. Shaposhnikova.

14. M. Putinar and N. Stylianopoulos, Finite-term relations for planar orthogonal polynomials, Complex Anal. Oper. Theory 1 (2007), no. 3, 447-456.

15. H. Render, Real Bargmann spaces, Fischer decompositions, and sets of uniqueness for polyharmonic functions, Duke Math. J. 142 (2008), no. 2, 313-352.

16. M. Sakai, Null quadrature domains, J. Analyse Math. 40 (1981), 144-154 (1982).

17. H. S. Shapiro, Unbounded Quadrature Domains, Complex analysis, I (College Park, Md., 1985-86), Lecture Notes in Math., vol. 1275, Springer, Berlin, 1987, pp. 287-331.

18. _ The Schwarz Function and its Generalization to Higher Dimensions, University of Arkansas Lecture Notes in the Mathematical Sciences, 9, John Wiley \& Sons Inc., New York, 1992, A Wiley-Interscience Publication.

19. P. K. Suetin, Polynomials Orthogonal over a Region and Bieberbach Polynomials, American Mathematical Society, Providence, R.I., 1974, Translated from the Russian by R. P. Boas.

20. G. Szegő, Orthogonal Polynomials, fourth ed., American Mathematical Society, Providence, R.I., 1975, American Mathematical Society, Colloquium Publications, Vol. XXIII.

Department of Mathematics and Statistics, University of South Florida, 4202 E. Fowler Avenue, PHY114, TAmpa, FL 33620-5700,, U.S.A.

E-mail address: dkhavins@cas.usf.edu

URL: http://math.usf.edu/faculty/dkhavinson/

Department of Mathematics and Statistics, University of Cyprus, P.O. Box 20537, 1678 Nicosia, Cyprus

E-mail address: nikos@ucy.ac.cy

$U R L:$ http://ucy.ac.cy/ nikos 